University of Wollongong

Research Online

Faculty of Engineering and Information

Faculty of Engineering and Information

Sciences - Papers: Part A

Sciences

$1-1-2013$

Electroactive polymers as soft robotic actuators: electromechanical modeling and identification

Rahim Mutlu

University of Wollongong, rm991@uowmail.edu.au

Gursel Alici

University of Wollongong, gursel@uow.edu.au

Weihua Li

University of Wollongong, weihuali@uow.edu.au

Follow this and additional works at: https://ro.uow.edu.au/eispapers

Part of the Engineering Commons, and the Science and Technology Studies Commons

Research Online is the open access institutional repository for the University of Wollongong. For further information contact the UOW Library: research-pubs@uow.edu.au 


\title{
Electroactive polymers as soft robotic actuators: electromechanical modeling and identification
}

\begin{abstract}
Biologically inspired robotic applications have recently received significant attention due to developments in novel materials and actuators with an operation principle similar to the natural muscles'. Electroactive polymer (EAP) actuators, also known as artificial muscles, possess extraordinary properties such as low efficiency consumption, compliance, bio-compatibility and ability to be miniaturized. Although several methodologies have been proposed for modeling and identification of their quasi-static bending behavior, a negligibly small attention has been given to their dynamic behavior. In this paper, we, therefore, report on their electromechanical modeling and parameter identification. We model the tri-layer EAP actuators as a soft robotic actuator consisting of a significant number of rigid links connected with compliant revolute joints. The experimental and numerical results presented suggest that the soft robotics approach is an effective way to model the EAP actuator and subsequently identify its dynamic parameters accurately. We have previously employed the same soft robotic approach to estimate the whole shape of the EAP actuator as a function of time.
\end{abstract}

\section{Keywords}

soft, electromechanical, polymers, modeling, electroactive, actuators, robotic, identification

\author{
Disciplines \\ Engineering | Science and Technology Studies
}

\section{Publication Details}

Mutlu, R., Alici, G. \& Li, W. (2013). Electroactive polymers as soft robotic actuators: electromechanical modeling and identification. $2013 \mathrm{IEEE} / \mathrm{ASME}$ International Conference on Advanced Intelligent Mechatronics (AIM) (pp. 1096-1101). United States: IEEE. 


\title{
Electroactive Polymers as Soft Robotic Actuators: Electromechanical Modeling and Identification
}

\author{
Rahim Mutlu, Gursel Alici and Weihua Li
}

\begin{abstract}
Biologically inspired robotic applications have recently received significant attention due to developments in novel materials and actuators with an operation principle similar to their natural counterparts, i.e. muscles. Electroactive polymer (EAP) actuators, also known as artificial muscles, possess extraordinary properties such as low efficiency consumption, compliance, bio-compatibility and ability to be miniaturized. Although several methodologies have been proposed for modeling and identification of their quasi-static bending behavior, a negligibly small attention has been given to their dynamic behavior. In this paper, we, therefore, report on their electromechanical modeling and parameter identification. We model the tri-layer EAP actuators as a soft robotic actuator consisting of a significant number of rigid links connected with compliant revolute joints. The experimental and numerical results presented suggest that the soft robotics approach is an effective way to model the EAP actuator and subsequently identify its dynamic parameters accurately. We have previously employed the same soft robotic approach to estimate the whole shape of the EAP actuator as a function of time.
\end{abstract}

\section{INTRODUCTION}

$\mathrm{S}$ OFT robotic devices based on highly flexible materials have gained significant momentum in the last decade due to their favorable characteristics such as compliance, compactness, ease of manufacturability, and being suitable to miniaturization. Of those materials used to establish artificial muscles exhibit natural muscle like behaviors due to their operation principle similar to real muscles $[1,2]$. The EAP actuators, the most popular of the artificial muscles, have several advantageous over other smart material actuators such as manufacturability in nano- and micro-size, small energy consumption, a high force output to weight ratio, biocompatibility, ability to operate in air and aqueous environments, compliance, and silent operation. EAP actuators have been proposed for applications including micro robotic gripping systems, energy converters, swimming devices, crawling robots, micro manipulators, stiffness regulators, motion converter mechanisms and many more [3-11]. The EAP actuators are especially suitable for

R. Mutlu (rm991@uowmail.edu.au) is with School of Mechanical, Materials and Mechatronic Engineering, University of Wollongong, NSW, 2522, Australia

G. Alici (Corresponding Author) is with School of Mechanical, Materials and Mechatronic Engineering and ARC Centre of Excellence for Electromaterials Science, University of Wollongong, NSW, 2522, Australia (ph:+6142214115,gursel@uow.edu.au)

W. Li (weihuali@uow.edu.au) is with School of Mechanical, Materials and Mechatronic Engineering, University of Wollongong, NSW, 2522, Australia biologically-inspired robotics -thanks to their natural muscle like working principles. Inspired from fish swimming, the EAP actuators were employed to establish swimming robots powered through their caudal fin for a nautical motion [6, 7]. Mutlu et al. [12] first time proposed the EAP actuators forming a helical shape from a planar spiral that is inspired from bacteria (i.e. E. coli) forming its flagella into a helical shape to swim. Sareh and Rossiter [13] have reported on bioinspired devices powered by ionic polymer metal composite (IPMC) actuators with which an atrioventricular cuspid valve and Vorticella campanula's retracting stalk motions are replicated.

A number of methodologies have been followed in the literature in order to model and analyze the EAP actuators' bending behaviors based on their chemical, electrical, mechanical properties and parameters. In most of these studies, the EAP actuators' bending behavior is analyzed using a cantilever-beam-bending approach in which an electrical stimulus is applied at the EAP actuator's fixed-end and its displacement is measured from the EAP actuator's free-end as an output using either a finite element method or black box approach (i.e. SISO based on a transfer function) [14-17]. Alici also applied the classical beam theory taking non-linear effects into account to estimate non-linear bending displacements of the PPy-EAP actuators [18]. However, these studies focus on quasi-static bending behavior of the EAP actuators. A more comprehensive model explaining their dynamic behavior will be crucial for the EAP actuators if they are to be employed in more advanced applications such as medical devices, micro robots, artificial organs/muscles and other bio-inspired applications. The dynamic model should not only estimate the EAP actuator's tip positions, but also be able to predict the whole bending behavior of the EAP actuator as a function of time.

One can follow several methods to obtain a dynamic model. In general, two methods are commonly used [19];

i. to identify a transfer function based on the system's input/output behavior,

ii. to establish a mathematical model using the NewtonEuler method, the Lagrangian or the Hamilton formulations.

While the first method is more suitable for modeling single input single output (SISO) systems, we use the second method in this study to model the Polypyrrole based tri-layer 
EAP (PPy-EAP) actuator. It is not straightforward to model and analyze the kinematic and dynamic behaviors of these cantilevered-type actuators as their operation principle based on the electrical, chemical mechanical parameters is not fully understood yet. The dynamic model developed in this paper is inspired by a soft robotic actuator or manipulator modeling approach in which the real structure is represented by a curve, known as the backbone curve. We incorporate a voltage-internal moment relation into the dynamic model to obtain an electromechanical model which can be used to understand the dynamic behavior of the PPy-EAP actuator for a given electrical input. After estimating the PPy-EAP actuator's whole shape configuration as a function of time using its hyper-redundant inverse kinematic model solved by an optimization (AngleOPT) method [20, 21], we experimentally identify the dynamic parameters of the PPyEAP actuator. We have experimentally validated the proposed electromechanical model which can be used for (i) identifying the stiffness and damping parameters of the actuator and (ii) controlling the actuator's whole shape deflection under an electrical stimulus without requiring any position/deflection feedback information.

\section{THE FABRICATION AND OPERATION PRINCIPLE OF EAP ACTUATORS}

The tri-layer laminated EAP actuators considered in this study are synthesized by following a number of steps. Pyrrole is used as polymerization monomer. Both sides of a porous layer (i.e. polyvinylidene fluoride, PVDF), as received (110 $\mu \mathrm{m}$ in thickness), were sputter coated with gold to produce a conductive surface for polymerization. Lithium triflouromethanesulfonimide (Li.TFSI) is used as the electrolytic ions, $\mathrm{Li}^{+} \mathrm{TFSI}^{-}$, which are stored in the PVDF layer. Polymerization solution was prepared containing $0.1 \mathrm{M}$ pyrrole monomer, $\mathrm{Li}^{+} \mathrm{TFSI}^{-}(0.1 \mathrm{M})$ and $1 \%$ water in propylene carbonate (PC). The gold coated PVDF was placed in the polymerization solution. Polypyrrole (PPy) layers were galvanostatically grown from the solution at a current density of $0.1 \mathrm{~mA} \mathrm{~cm}{ }^{-2}$ for 12 hours on the gold coated PVDF. 12-hr polymerization process provides $\sim 30$ $\mu \mathrm{m}$ thickness of a PPy layer on each side of the gold coated PVDF. The PPy based EAP actuator was cut from the bulk sheet fabricated which we call the PPy-EAP actuator throughout the paper unless otherwise stated. The PPy-EAP actuator's laminated structure and operation principle are depicted in Figure 1.

The PPy-EAP actuator's operation principle is based on the energy conversion from an electrochemical process to a mechanical output. An electrical input applied to the PPy layers stimulates counter-ions to move in and out of the PPy layers. While the positively charged polymer layer is oxidized, the negatively charged polymer layer is reduced. The TFSI $^{-}$anions move from electrolyte into the positively charged PPy layer and an opposite reaction happens in the other PPy layer in order to neutralize the charge in the PPy layers. This ion transfer causes a volume expansion in the positively charged PPy layer and a volume contraction in the other PPy layer. This electro-chemo-mechanical process therefore generates a mechanical bending in the PPy-EAP actuator, as illustrated in Figure 1.

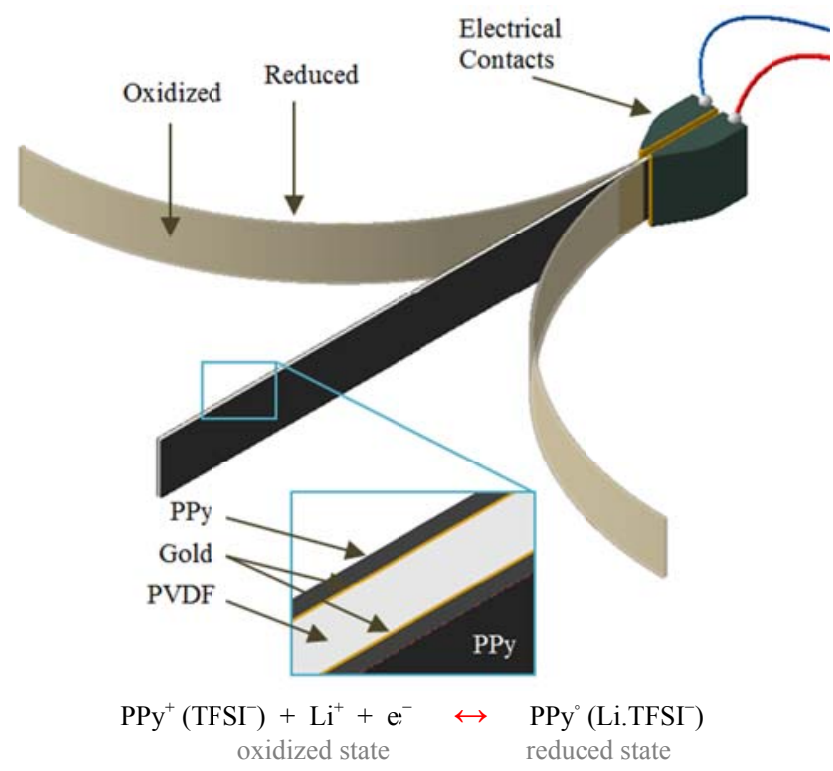

Figure 1. Structure and operation principle of the PPy-EAP actuator.

\section{Soft Robotic Kinematic Model Of PPy-EAP ACTUATOR}

Classical beam theories can be applied to structures with constant material properties such as a constant elasticity modulus. Cantilevered EAP actuators have been modeled and analyzed using a constant elasticity modulus assumption [14-17]. The constant elasticity modulus assumption can be applied to the EAP actuator bending in a linear range. This assumption amputates the applicability of the EAP actuators due their highly non-linear strain or deflection. Further, the classical beam theories assume that the beam deflection or the displacement output is less than $15 \%$ of the actuator beam length. As reported before, the cantilevered EAP actuators can generate tip deflections as high as $50 \%$ of their length [22]. With this in mind, one should follow a different methodology taking active material properties into account rather than using a constant property assumption, and linear actuator deflection output. We use soft robotic structure modeling approach which assumes an imaginary curve (i.e. so called backbone curve) passing through the geometric centers of the discretized cross-sections of the real soft robotic structure. In our previous papers [12, 20, 21], we demonstrated that the soft robotic modeling approach can accurately estimate the highly non-linear whole shape bending behavior of the PPy-EAP actuators. The soft robotic modeling approach has been reported on some bio-inspired robotic manipulators including snake-like robot [23], octopus arm [24] and elephant trunk robot [25, 26]. Mathematically expressing the backbone curve of the soft robotic structure, which becomes its kinematic model, is straightforward. However, obtaining solutions to this soft 
robotic kinematic model can be problematic. Conventional methods such as differential kinematics based on the Jacobian matrix cannot be employed due to numerical instabilities close to kinematically singular configurations and inversion problems associated with non-square Jacobian matrix of the soft robotic kinematic model [27]. Mode shape and optimization methods can serve well to solve inverse kinematics of the soft robotic structure modeled using the backbone curve approach [23, 28-30]. Mode shape method is limited to the case in which movements of the soft robotic structure are predetermined for certain modes which should be matched by the backbone curvature of the soft robotic structure. These mode shapes are then incorporated into the forward kinematic model. Mode shape method is more suitable for soft robotic structures where feedback control from joints is possible. However, the purpose of the soft robotic kinematic modeling in this study is to estimate the shape that the real soft robotic structure (i.e. PPy-EAP actuator in this case) forms. Optimization methods are therefore more suitable to solve inverse kinematics of the PPy-EAP actuator using the backbone curve topology. Further, optimization based approaches do not require mathematical manipulations, provided that appropriate joint constraints are imposed on the inverse kinematic solutions. We construct an inverse kinematic model of the PPy-EAP actuator and then solve this inverse kinematic model by employing a non-linear constraint optimization method, which we call the AngleOPT, to estimate all configurations of the actuator throughout its movement under an electrical input. While details of the soft robotic kinematic model and solving its inverse kinematics can be found in [20,21], we provide some brief information here for the sake of completeness.

The backbone curve of the PPy-EAP actuator operating in planar conditions (the actuator operates in horizontal plane) can be defined as, with respect to the fixed-end of the actuator, as shown in Figure 2,

$$
r(\sigma, t)=\left[r_{x}(\sigma, t) r_{y}(\sigma, t)\right]^{T}
$$

where $r(\sigma, t):[0, L] \rightarrow R^{2}$ assigns a position vector in the Euclidean space to each link (i.e. discretized section of the backbone curve of the soft robotic structure) parameter, $\sigma \in$ $[0, \mathrm{~L}]$ in an instant time, $\mathrm{t}[12,31]$. $\mathrm{L}$ is the overall length of the backbone curve.

Inverse kinematic solutions of the PPy-EAP actuator's hyper-redundant kinematic model are obtained by converting its kinematic model into a non-linear constrained optimization problem with an adaptive boundary-constraint approach. In other words, the PPy-EAP actuator's shape variation during its operation becomes a shape estimation problem solved by optimization. The objective function given below is based on the tip coordinates, $\left[\begin{array}{ll}X & Y\end{array}\right]^{T}$, of the PPy-EAP actuator;

$$
\min _{\theta} f=\min _{\theta}\left(\sum_{\mathrm{i}=1}^{\mathrm{n}}\left[\begin{array}{l}
\mathrm{r}_{\mathrm{x}}\left(\sigma_{\mathrm{i}}, \mathrm{t}\right) \\
\mathrm{r}_{\mathrm{y}}\left(\sigma_{\mathrm{i}}, \mathrm{t}\right)
\end{array}\right]-\left[\begin{array}{l}
X \\
Y
\end{array}\right]_{i}\right)
$$

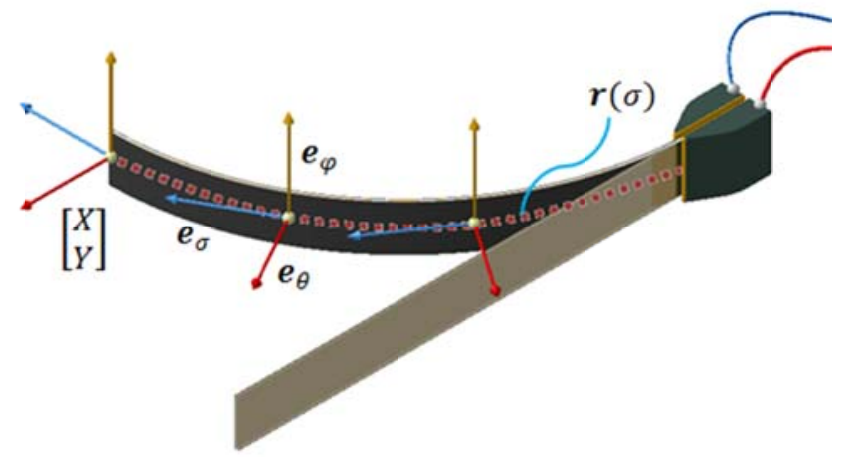

Figure 2. An EAP actuator 's configuration and its soft robotic model representation defined by' its backbone curve, $\boldsymbol{r}(\sigma)$.

As the PPy-EAP actuator's actuation configuration is cantilevered, the models are developed in 2D. The shape estimation problem (Eq.2) based on the kinematic model is subjected to physical constraints of the PPy-EAP actuator in operation. $r_{x}\left(\sigma_{i}, t\right)$ and $r_{y}\left(\sigma_{i}, t\right)$ are the position coordinates of each section of the PPy-EAP actuator, $t$ is time, and $n$ is the number of links.

\section{Soft Robotic Electromechanical Model of PPy- EAP ACTUATOR}

The electromechanical model of the PPy-EAP actuator is obtained by incorporating its input voltage - internal moment relation into its dynamic model. The dynamic model of the PPy-EAP actuator is obtained by employing the Lagrange equations to the soft robotic structure. The kinetic energy of $i$-th link on the backbone curve is

$$
K_{i}(\theta, t)=\frac{1}{2}\left\{m_{i}\left[\left(\frac{\partial x_{i}}{\partial t}\right)^{2}+\left(\frac{\partial y_{i}}{\partial t}\right)^{2}\right]+J_{i \theta}\left[\left(\frac{\partial \theta_{i}}{\partial t}\right)^{2}\right]\right\}
$$

where $\mathrm{m}_{\mathrm{i}}$ is the mass and $\mathrm{J}_{\mathrm{i}}$ is the inertia tensor of the discretized section measured from the center of mass of the section which is described as

$$
J_{i \theta}=\left[\begin{array}{ll}
I_{x x} & I_{x y} \\
I_{y x} & I_{y y}
\end{array}\right]
$$

The total kinetic energy is given by

$$
\mathcal{K}(\mathrm{t}) \triangleq \sum_{1}^{\mathrm{n}} \mathrm{K}_{\mathrm{i}}(\theta, \mathrm{t})
$$

The potential energy of the PPy-EAP actuator consists of two components; elastic potential energy and gravitational potential energy. The elastic potential energy of $i$-th discretized section on the backbone curve is

$$
\mathrm{P}_{\mathrm{bi}}(\theta, \mathrm{t})=\frac{1}{2}\left[\mathrm{k}_{\theta \mathrm{i}}\left(\theta_{\mathrm{i}}-\theta_{\mathrm{i}-1}\right)^{2}\right]
$$

where $\mathrm{k}_{\theta \mathrm{i}}$ is the stiffness constant for each generalized coordinate, $\theta_{i}$. Elastic bending property of the PPy-EAP 
actuator is represented by the stiffness constants. The total elastic potential energy is given by

$$
\mathcal{P}_{\mathrm{b}}(\mathrm{t}) \triangleq \sum_{1}^{\mathrm{n}} \mathrm{P}_{\mathrm{bi}}(\theta, \mathrm{t})
$$

where $\mathrm{P}_{\mathrm{bi}}(\theta, \varphi, t)$ is the elastic energy of the $i$-th discretized section on the backbone curve of the EAP actuator. The gravitational potential energy of each discretized section is given by

$$
\mathrm{P}_{\mathrm{gi}}(\theta, \mathrm{t})=\mathrm{m}_{\mathrm{i}} \mathrm{g} \mathrm{z}_{\mathrm{i}}(\theta, \varphi, \mathrm{t})
$$

where $g$ is the gravitational acceleration, $z_{i}(\theta, \varphi, t)$ is the vertical distance between the centre of mass of $i$-th discretized section and the base plane. The total gravitational potential energy is obtained as

$$
\mathcal{P}_{\mathrm{g}}(\mathrm{t}) \triangleq \sum_{1}^{\mathrm{n}} \mathrm{P}_{\mathrm{gi}}(\theta, \varphi, \mathrm{t})
$$

If the EAP actuator operates in horizontal plane where vertical distance of discretized sections along the backbone curve do not change, the potential energy of the EAP actuator depends only on its elastic bending property. The Lagrangian is therefore given in the following form [32]

$$
\mathcal{L}(\mathrm{t})=\mathcal{K}(\mathrm{t})-\mathcal{P}(\mathrm{t})
$$

where $\mathcal{K}(\mathrm{t})$ is the total kinetic energy and $\mathcal{P}(\mathrm{t})$ is the total potential energy of the EAP actuator. Using the Lagrangian, the motion equations are obtained from

$$
\left\{\frac{\mathrm{d}}{\mathrm{dt}} \frac{\partial \mathcal{L}}{\partial \dot{\mathrm{q}}_{\mathrm{i}}}-\frac{\partial \mathcal{L}}{\partial \mathrm{q}_{\mathrm{i}}}\right\}=\left\{\mathcal{J}_{\mathrm{i}}-\mathrm{b} \dot{\mathrm{q}}_{\mathrm{i}}\right\}, \quad \mathrm{i}=1,2, \ldots, \mathrm{n}
$$

where $q_{i}$ represents generalized coordinates at each joint. $\mathcal{T}_{\mathrm{i}}$ is the torque (i.e. internal bending moment) at $i$-th joint. b indicates the damping coefficient in the rotational generalized coordinates.

As the purpose of the dynamic modeling of the EAP actuator is to estimate the actuator's dynamic behavior for applied electrical input, a relation is required between mechanical load and input voltage. The mechanical load is generated by a series of electrochemical reactions in the EAP actuator. The electrical input stimulates volume change in the active polymer layer and this volume change (i.e. strain) creates stress field (i.e. mechanical load or pressure) $[9,10]$. This mechanical load is then used to calculate internal moments or torques in the dynamic model obtained. The internal moment for each joint in the hyper-redundant model of the EAP actuator can be expressed as a function of time and input voltage [18];

$$
\begin{gathered}
\mathrm{p}_{\text {load }}(\mathrm{t}, \mathrm{V})=-\left(\frac{8}{3}\right) *\left(\frac{\mathrm{F}_{\text {blocking }}(\mathrm{t}, \mathrm{V})}{\mathrm{L}}\right) \\
\mathrm{M}_{\mathrm{i}}=\sum_{\mathrm{i}=1}^{\mathrm{n}} \mathrm{p}_{\text {load }} \frac{\mathrm{i}^{2} \mathrm{l}}{2} \\
\mathcal{T}_{\mathrm{i}}=\mathrm{M}_{\mathrm{i}}-\mathrm{M}_{\mathrm{i}-1}
\end{gathered}
$$

where $F_{\text {blocking }}$ is the blocking force measured experimentally, $L$ and $l$ are the overall length and each link length of the EAP actuator, respectively. The electromechanical equations can be written in a matrixvector form by employing Eqs. 11-14;

$$
\begin{array}{r}
\mathcal{T}(\mathrm{t})=\mathrm{D}(\mathrm{q}(\mathrm{t})) \ddot{\mathrm{q}}(\mathrm{t})+\mathrm{H}(\mathrm{q}(\mathrm{t}), \dot{\mathrm{q}}(\mathrm{t}))+\mathrm{C}(\mathrm{q}(\mathrm{t})) \\
\text { V. EXPERIMENTAL RESULTS AND DISCUSSION }
\end{array}
$$

The PPy-EAP actuator used in the experiments was cut into the dimensions of $20 \times 3 \times 0.17 \mathrm{~mm}$, length, width and thickness, respectively. Although there is no restriction on the number of links in the soft robotic actuator model development, the kinematic and dynamic models are developed as 16-Dof hyper-redundant actuator (i.e. 16 serially connected rigid links with compliant and damped joints). The higher is the number of the links in the soft robotic actuator model; the better is the shape correspondence between the real EAP actuator and the model. Of course, the computational cost will increase as the number of links increases in the models.

The EAP actuator's elastic behaviors are conditiondependent; electrical input, ion diffusion, the geometry, porosity in the tri-layers, and conductivity of the active layers, electrolyte conductivity and its molarity change the EAP actuator's elastic behavior. Joint stiffness and damping parameters should be estimated dynamically [33-35]. Joint stiffness and damping values are obtained by employing a direct dynamic parameter identification method in which the identification is performed using experimental data. We employ a non-linear least-square formulation to identify the joint stiffness and damping parameters by minimizing the error between experimental and simulation results as formulated below;

$$
\vartheta=\arg \min _{\vartheta} \sum_{\mathrm{t}=1}^{\mathrm{N}} \sum_{\mathrm{k}=1}^{\text {DoF }} \frac{\left(\mathcal{T}_{\mathrm{k}}(\mathrm{t})-\mathrm{W}_{\mathrm{k}}\left[\mathrm{q}_{\mathrm{k}}(\mathrm{t}), \dot{\mathrm{q}}_{\mathrm{k}}(\mathrm{t}), \ddot{\mathrm{q}}_{\mathrm{k}}(\mathrm{t}), \vartheta\left(\mathrm{k}_{\mathrm{k}}, \mathrm{b}_{\mathrm{k}}\right)\right]\right)^{2}}{\sigma^{2}}
$$

where $\mathrm{W}_{\mathrm{k}}$ is the $k$-th element of the right side of the Eq.15, $\sigma$ is the standard deviation and $\vartheta$ represents unknown parameters (i.e. joint stiffness and damping values).

The key components of the experimental setup are presented in Figure 4 in three main groups; actuation and measurement (i.e. tip displacement measurement by an image processing and force measurement system), inverse kinematic shape estimation, and electromechanical model validation. The electrical input signals were generated using a SIMULINK program and passed through an USB-type NIDAQ card (NI USB-6251) to a potentiostat. The electrical inputs were applied to the PPy-EAP actuator using a gold coated clamp. The blocking force data were obtained using a dual-mode lever arm (Aurora Scientific, 300C-LR) system. The motion of the PPy-EAP actuator was recorded using a digital camera (Nikon D5100). The PPy-EAP actuator was stimulated from its neutral state (i.e. straight) to a fully-bent state. The PPy-EAP actuator was tested by applying input 
voltages from 0.25 to $1.00 \mathrm{~V}$ with $0.25 \mathrm{~V}$ increment at each test. The tip position data of the PPy-EAP actuator for each test was obtained from recorded videos by employing an image processing algorithm. The tip position data is then used to estimate the whole shape of the PPy-EAP actuator by employing the AngleOPT which is used to solve its hyper-redundant inverse kinematic model under a set of the boundary conditions and constraints. Inverse kinematic solutions (i.e. shape variation of the PPy-EAP actuator) are used in the dynamic parameter identification procedure in order to obtain stiffness and damping values for each joint of the PPy-EAP actuator's soft robotic electromechanical model, as presented in Figure 3. It must be noted that the joint stiffness and damping parameters change with the input voltage. Calculating the stiffness or modulus of elasticity of smart actuators is a challenging task. With the proposed modeling and parameter estimation method, this can be done effectively. The electromechanical model is validated using these joint stiffness and damping parameters, and the internal moment relations (Eqs. 12-14). The motion equations, i.e. the dynamic model, for the PPy-EAP actuator are solved numerically and the results are exhibited in Figure 5 , with the real PPy-EAP actuator is at the background which shows an excellent match between the numerical and experimental results. To demonstrate the efficacy of the model in estimating the tip deflections, a new set of experimental results were generated to compare them with the numerical results obtained from the solution of the electromechanical model with the identified parameters.

The soft robotic electromechanical model we have established for the EAP actuators uses the backbone curve approach for kinematic model, and Lagrange equations for its dynamic model. This proposed methodology estimates the highly non-linear bending behavior of the EAP actuators accurately. The numerical solutions from the model show an excellent correlation with the experimental results. This methodology is quite effective in not only estimating highly flexible EAP actuator bending behaviors kinematically and dynamically, but also identifying the dynamic parameters of the EAP actuator.
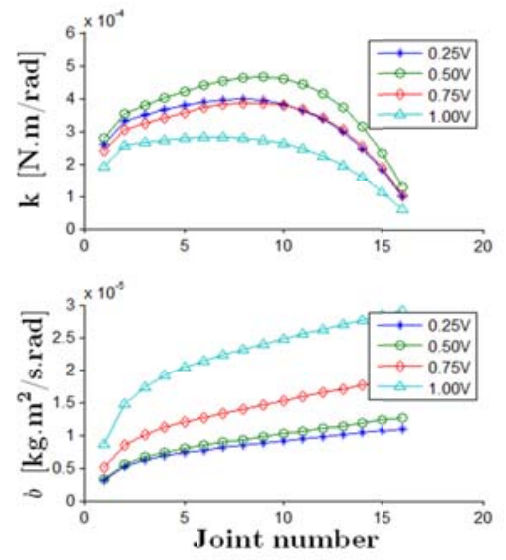

Figure 3. Joint stiffness and damping values of the PPy-EAP actuator under $0.25-1.00 \mathrm{~V}$ (color print).

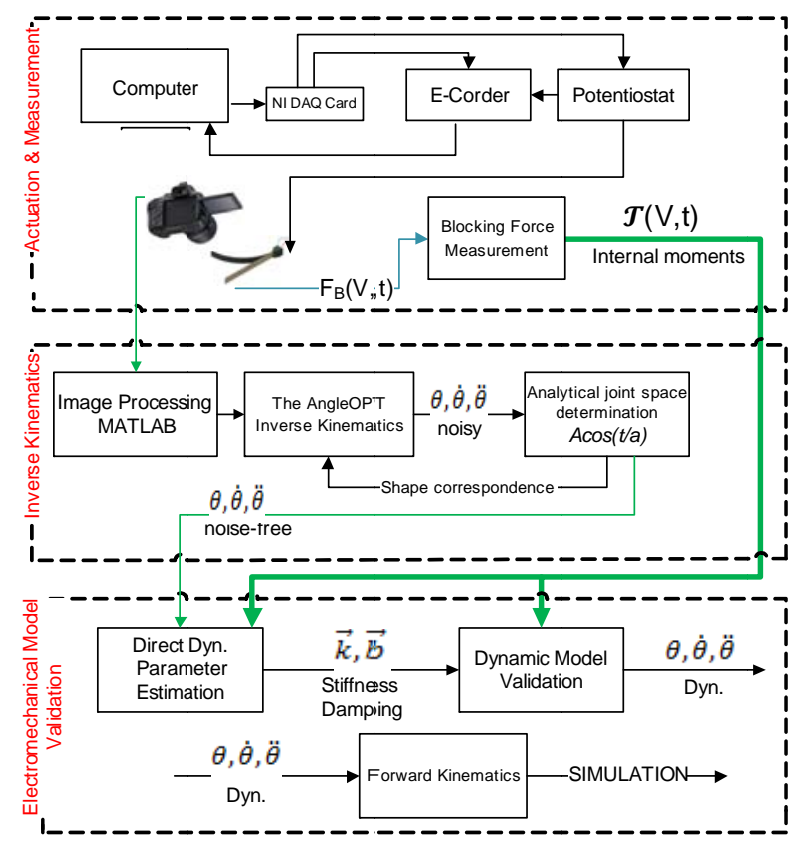

Figure 4. Schematic of the experimental setup and the electromechanical model validation.
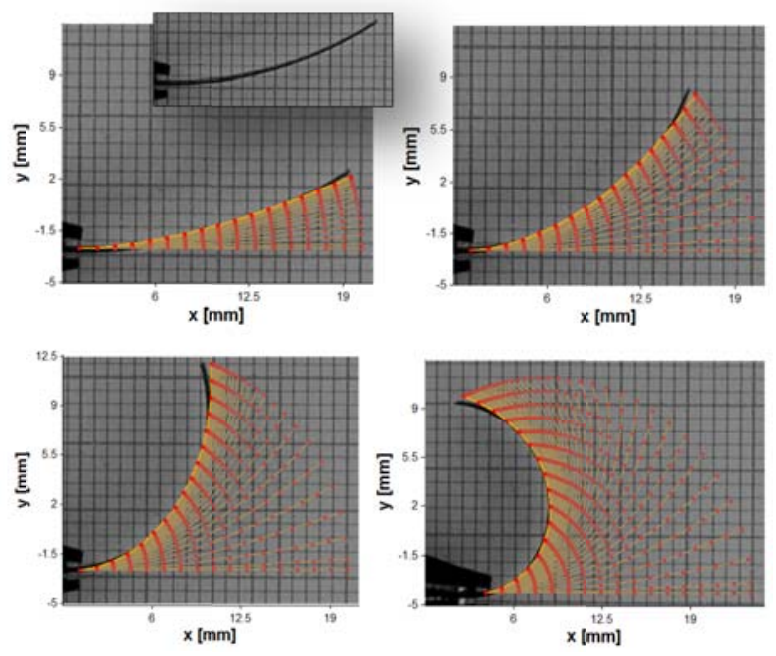

Figure 5. Comparison between the 16-dof electromechanical model (red dotted orange curve) and the PPy-EAP actuator (black thick curve at the background image) under $0.25-1.00 \mathrm{~V}$ (color print).

\section{CONCLUSION AND FUTURE WORK}

We have demonstrated an effective methodology to model and identify the dynamic behavior of the PPy based tri-layer laminated EAP actuators which we have been modeled as a soft robotic actuator with a continuous configuration. The proposed methodology is not only a reliable tool for estimating the EAP actuators' highly nonlinear dynamic bending behaviors but also obtaining their elastic parameters directly from the experimental data. The soft robotic electromechanical actuator model gives us a more realistic insight about the EAP actuators' bending 
behavior and enables us to control their displacement and force output for given electrical inputs (i.e. less than $1.00 \mathrm{~V}$ ) in more advanced applications including active compliant mechanisms, biomedical applications and bio-inspired micro-robotic devices.

Future work includes validating the proposed modeling and identification methodology for the EAP actuators with different geometric parameters and demonstrating the relationship between their geometric and dynamic parameters. We will also test this soft robotic electromechanical model for the EAP actuators with various external loading conditions. This model will also be used to predict the dynamic behavior of a multi-stable linear actuation mechanism [10] and will serve well for its design optimization.

\section{ACKNOWLEDGEMENTS}

This work was supported in part by the ARC Centre of Excellence for Electromaterials Science (CE0561616) and an ARC Discovery project (Grant No. DP0878931).

\section{REFERENCES}

[1] B. Tondu, "Artificial Muscles for Humanoid Robots," in Humanoid Robots, Human-like Machines, M. Hackel, Ed., ed, 2007.

[2] Y. Bar-Cohen, Electroactive Polymer (EAP) Actuators as Artificial Muscles: Reality, Potential, and Challenges, 2nd ed. Bellingham: SPIE Press, 2004.

[3] G. Alici and N. N. Huynh, "Performance Quantification of Conducting Polymer Actuators for Real Applications: A Microgripping System," Mechatronics, IEEE/ASME Transactions on, vol. 12, pp. 73-84, 2007.

[4] G. Alici, V. Devaud, P. Renaud, and G. Spinks, "Conducting polymer microactuators operating in air," Journal of Micromechanics and Microengineering, vol. 19, p. 025017, 2009.

[5] Y. Wu, G. Alici, G. M. Spinks, and G. G. Wallace, "Fast trilayer polypyrrole bending actuators for high speed applications," Synthetic Metals, vol. 156, pp. 1017-1022, 2006.

[6] G. Alici, G. Spinks, N. N. Huynh, L. Sarmadi, and R. Minato, "Establishment of a biomimetic device based on tri-layer polymer actuators--propulsion fins," Bioinspiration \& Biomimetics, vol. 2, pp. $18-30,2007$.

[7] S. McGovern, G. Alici, V.-T. Truong, and G. Spinks, "Finding NEMO (novel electromaterial muscle oscillator): a polypyrrole powered robotic fish with real-time wireless speed and directional control," Smart Materials and Structures, vol. 18, p. 095009, 2009.

[8] G. Alici and D. Gunderson, "A bio-inspired robotic locomotion system based on conducting polymer actuators," in Advanced Intelligent Mechatronics, 2009. AIM 2009. IEEE/ASME International Conference on, 2009, pp. 998-1004.

[9] R. Mutlu and G. Alici, "Artificial muscles with adjustable stiffness," Smart Materials and Structures, vol. 19, p. 045004, 2010.

[10] R. Mutlu and G. Alici, "A Multistable Linear Actuation Mechanism Based on Artificial Muscles," Journal of Mechanical Design, vol. 132, p. 111001, 2010.

[11] G. Alici, G. M. Spinks, J. D. Madden, W. Yanzhe, and G. G. Wallace, "Response Characterization of Electroactive Polymers as Mechanical Sensors," Mechatronics, IEEE/ASME Transactions on, vol. 13, pp. 187-196, 2008

[12] R. Mutlu, G. Alici, and L. Weihua, "Kinematic modeling for artificial flagellum of a robotic bacterium based on electroactive polymer actuators," in Advanced Intelligent Mechatronics (AIM), 2011 IEEE/ASME International Conference on, 2011, pp. 440-445.

[13] S. Sareh and J. Rossiter, "Kirigami artificial muscles with complex biologically inspired morphologies," Smart Materials and Structures, vol. 22, p. 014004, 2013.
[14] G. Alici, P. Metz, and G. M. Spinks, "A methodology towards geometry optimization of high performance polypyrrole (PPy) actuators," Smart Materials and Structures, vol. 15, p. 243, 2006.

[15] S. John, G. Alici, and C. Cook, "Frequency response of polypyrrole trilayer actuator displacement," in Electroactive Polymer Actuators and Devices (EAPAD), San Diego, California, 2008, p. 69271T.

[16] S. W. John, G. Alici, and C. D. Cook, "Validation of Resonant Frequency Model for Polypyrrole Trilayer Actuators," Mechatronics, IEEE/ASME Transactions on, vol. 13, pp. 401-409, 2008.

[17] S. W. John, G. Alici, and C. D. Cook, "Towards the position control of conducting polymer trilayer bending actuators with integrated feedback sensor," in Advanced Intelligent Mechatronics, 2009. AIM 2009. IEEE/ASME International Conference on, 2009, pp. 65-70.

[18] G. Alici, "An effective modelling approach to estimate nonlinear bending behaviour of cantilever type conducting polymer actuators," Sensors and Actuators B: Chemical, vol. 141, pp. 284-292, 2009.

[19] R. C. Dorf and R. H. Bishop, "Mathematical models of systems," in Modern control systems, 11th ed NJ: Pearson Prentice Hall, 2008, pp. 41-143.

[20] R. Mutlu, G. Alici, and L. Weihua, "Kinematic analysis of electroactive polymer actuators as soft and smart structures with more DoF than inputs," in Advanced Intelligent Mechatronics (AIM), 2012 IEEE/ASME International Conference on, 2012, pp. 484-489.

[21] R. Mutlu, G. Alici, and W. Li, "An effective methodology to solve inverse kinematics of electroactive polymer actuators modelled as active and soft robotic structures," Mechanism and Machine Theory, 2013, http://dx.doi.org/10.1016/j.mechmachtheory.2013.04.005.

[22] P. G. A. Madden, "Development and modeling of conducting polymer actuators and the fabrication of a conducting polymer based feedback loop," Ph.D. thesis, Dept. of Mechanical Engineering, Massachusetts Institute of Technology, 2003.

[23] S. Hirose and M. Mori, "Biologically Inspired Snake-like Robots," in Robotics and Biomimetics, 2004. ROBIO 2004. IEEE International Conference on, 2004, pp. 1-7.

[24] Y. Yekutieli, R. Sagiv-Zohar, R. Aharonov, Y. Engel, B. Hochner, and T. Flash, "Dynamic Model of the Octopus Arm. I. Biomechanics of the Octopus Reaching Movement," Journal of Neurophysiology, vol. 94, pp. 1443-1458, 2005.

[25] M. W. Hannan and I. D. Walker, "Novel Kinematics for Continuum Robots," in Advances in Robot Kinematics, J. Lenarčič and M. M. Stanišić, Eds., ed: Springer Netherlands, 2000, pp. 227-238.

[26] M. W. Hannan and I. D. Walker, "Kinematics and the Implementation of an Elephant's Trunk Manipulator and Other Continuum Style Robots," Journal of Robotic Systems, vol. 20, pp. 45-63, 2003.

[27] G. Alici, "An inverse position analysis of five-bar planar parallel manipulators," Robotica, vol. 20, pp. 195-201, 2002.

[28] H. Mochiyama, E. Shimemura, and H. Kobayashi, "Shape correspondence between a spatial curve and a manipulator with hyper degrees of freedom," in Intelligent Robots and Systems, 1998. Proceedings., 1998 IEEE/RSJ International Conference on, 1998, pp. 161-166.

[29] G. S. Chirikjian, "A continuum approach to hyper-redundant manipulator dynamics," in Intelligent Robots and Systems '93, IROS '93. Proceedings of the 1993 IEEE/RSJ International Conference on, 1993, pp. 1059-1066.

[30] G. S. Chirikjian, "Inverse Kinematics of Binary Manipulators Using a Continuum Model," J. Intell. Robotics Syst., vol. 19, pp. 5-22, 1997.

[31] V. A. Toponogov, "Theory of Curves in Three-dimensional Euclidean Space and in the Plane," in Differential Geometry of Curves and Surfaces, V. Rovenski, Ed., ed: Birkhäuser Boston, 2006, pp. 1-63.

[32] A. A. Shabana, "Analytical techniques," in Dynamics of Multibody Systems, 3rd ed NY: Cambridge University Press, 2005, pp. 85-158.

[33] K. Kozłowski, "Experimental identification of robot dynamic parameters," in Modelling and Identification in Robotics, ed NY: Springer-Verlag, 1998, pp. 131-188.

[34] P. Depince, "Parameters identification of flexible robots," in Robotics and Automation, 1998. Proceedings. 1998 IEEE International Conference on, 1998, pp. 1116-1121.

[35] S. Hai-Jun, "A pseudo-rigid-body 3R model for determining large defection of cantilever beams subject to tip loads," ASME Journal of Mechanisms and Robotics, vol. 1, p. 021008, 2009. 